\title{
1 Mechanisms of transforming DNA uptake to the 2 periplasm of Bacillus subtilis
}

3

4

5 Jeanette Hahna, Micaela DeSantisa, David Dubnaua\#

6

7 aPublic Health Research Institute and Department of Microbiology, Biochemistry

8 and Molecular Genetics, New Jersey Medical School, Rutgers University

13 Jeanette Hahn: Public Health Research Institute, New Jersey Medical School,

14 Rutgers University, hahnje@njms.rutgers.edu

15

16 Micaela De Santis: Public Health Research Institute, New Jersey Medical School,

17 Rutgers University, md1203@njms.rutgers.edu

18

19 David Dubnau: Public Health Research Institute and Department of Microbiology,

20 Biochemistry and Molecular Genetics, New Jersey Medical School, Rutgers

21 University, dubnauda@njms.rutgers.edu

22

23 Address correspondence to David Dubnau, dubnauda@njms.rutgers.edu

Word count abstract: 194

Word count text: 5398 


\section{ABSTRACT}

35 We demonstrate here that the acquisition of DNAase resistance by transforming

36 DNA, often assumed to indicate transport to the cytoplasm, actually reflects

37 uptake to the periplasm, requiring a re-evaluation of conclusions about the roles

38 of several proteins in transformation. The new evidence suggests that the

39 transformation pilus is needed for DNA binding to the cell surface near the cell

40 poles and for the initiation of uptake. The cellular distribution of the membrane-

41 anchored ComEA of $B$. subtilis does not noticeably change during DNA uptake as

42 does the unanchored ComEA of Vibrio and Neisseria. Instead, our evidence

43 suggests that ComEA stabilizes the attachment of transforming DNA at localized

44 regions in the periplasm and then mediates uptake, probably by a Brownian

45 ratchet mechanism. Following that, the DNA is transferred to periplasmic portions

46 of the channel protein ComEC, which plays a previously unsuspected role in

47 uptake to the periplasm. We show that the transformation endonuclease NucA

48 also facilitates uptake to the periplasm and that the previously demonstrated role

49 of ComFA in the acquisition of DNAase resistance actually derives from the

50 instability of ComGA when ComFA is deleted. These results prompt a new

51 understanding of the early stages of DNA uptake for transformation.

53 IMPORTANCE Transformation is a widely distributed mechanism of bacterial

54 horizontal gene transfer that plays a role in the spread of antibiotic resistance and

55 virulence genes and more generally in evolution. Although transformation was

56 discovered nearly a century ago and most, if not all of the proteins required have

57 been identified in several bacterial species, much remains poorly understood

58 about the molecular mechanism of DNA uptake. This study uses epifluorescence

59 microscopy to investigate the passage of labeled DNA into the compartment

60 between the cell wall and the cell membrane of Bacillus subtilis, a necessary early

61 step in transformation. The roles of individual proteins in this process are

62 identified, and their modes of action are clarified. 


\section{INTRODUCTION}

65 Transformation, the ability to internalize high molecular weight

66 environmental DNA, is widespread among bacteria and is a major mechanism of

67 horizontal gene transfer (reviewed in (1-3)). Several proteins that mediate

68 transformation are widely conserved, suggesting that the core mechanism is of

69 ancient origin. Although most of these proteins were identified more than three

70 decades ago in a genetic screen $(4,5)$, much remains to be learned of the

71 mechanisms that mediate the transfer of DNA across the multiple layers that

72 comprise the bacterial surface barrier.

73 In the Gram-negative bacteria Vibrio cholerae and Neisseria gonorrhoeae, a

74 type 4 pilus (t4 pilus) snares transforming DNA (tDNA) and retracts, threading

75 the tDNA through a pore in the outer membrane formed by a ring of secretin

76 subunits and thus into the periplasm (6-9). There, the tDNA encounters the DNA-

77 binding protein ComEA, a periplasmic protein that serves as a Brownian ratchet

78 (10), preventing back diffusion of the tDNA which consequently is believed to

79 accumulate in the periplasm before crossing the inner cell membrane $(7-9,11$,

80 12). In these bacteria, ComEA diffuses to the site of transforming tDNA uptake

81 where it concentrates dramatically as it binds to inward-diffusing tDNA.

82 Gram-positive bacteria lack an outer membrane. Instead, Bacillus subtilis, the

83 subject of the present study, is surrounded by a formidable cell wall, roughly 30-

$8435 \mathrm{~nm}$ in thickness, enclosing a 20-40 $\mathrm{nm}$ thick periplasm, defined as the

85 compartment between the wall and the cell membrane (13-15). This

86 compartment is probably gel-like, containing proteins, small molecules and

87 membrane-anchored lipoteichoic acid $(14,16)$, all of which may restrict diffusion

88 by molecular crowding. The wall of $B$. subtilis is a complex structure, including

89 peptidoglycan, proteins and wall teichoic acid (14). These differences in the outer

90 layers of Gram-positive and Gram-negative bacteria suggest consequent

91 differences in their mechanisms of DNA uptake to the periplasm. In fact, wall

92 teichoic acid, which is exclusive to the Gram positives, has been proposed to play

93 a role in DNA binding to competent cells (17). Also suggestive of such differences

94 is that B. subtilis ComEA is an integral membrane protein with its C-terminal DNA 
95 binding motifs exposed to the periplasm $(18,19)$ as opposed to the unanchored

96 ComEA proteins of $V$. cholerae and N. gonorrhoeae that can diffuse within the 97 periplasm.

98 Transformable Gram-positive bacteria, like their Gram-negative counterparts, 99 encode t4pilus-like proteins that form filamentous structures. In Streptococcus 100 pneumoniae, these proteins assemble fibers that can extend several micrometers 101 into the extracellular environment and can bind DNA (20-22). No such extended 102 structure has been described in B. subtilis, which instead seems to form a shorter 103 "pseudopilus" that is probably long enough to traverse the wall (23). This 104 transformation pseudopilus (tpilus) is encoded by the 7 genes of the comG operon 105 and also requires $\operatorname{com} C$ and $b d b D C$ for its construction (23-28). ComC is a 106 membrane peptidase that processes ComGC, the major pre-pilin subunit as well 107 as several minor pre-pilins, while $\mathrm{BdbD}$ and $\mathrm{BdbC}$ are thiol-disulfide 108 oxidoreductases that introduce intramolecular and intermolecular disulfide 109 bonds into ComGC and ComEC, the latter a component of the transformation 110 permease (29). comGA, the first gene of the comG operon, encodes a traffic ATPase 111 that is required for tpilus assembly (30). By analogy with $V$. cholerae (6) it is likely 112 that the Gram-positive tpili retract to bring DNA into the periplasmic 113 compartment. Indeed, a recent study shows convincingly that the pili of $S$. 114 pneumoniae extend and rapidly retract (22), although no such evidence has been 115 reported for $B$. subtilis.

116 In all transformable bacteria, transport across the cell membrane is 117 accomplished with the participation of ComEC, which is believed to form a 118 channel for the passage of DNA. In B. subtilis and S. pneumoniae, transport also 119 requires the membrane associated ComFA ATPase and ComFC, its binding partner 120 (31-35). During transport, one strand of the transforming tDNA is degraded while 121 the transforming strand enters the cytosol (36), where it associates with DNA 122 binding proteins and recombines with homologous DNA sequences to yield a 123 transformant.

124 In both Gram-negative and Gram-positive bacteria, tDNA becomes resistant to 125 added DNAase I (hereafter DNAase) during an early step in transformation. In the 
126 Gram-negatives, DNAase-resistance signals that the tDNA has breached the outer

127 membrane. In Gram-positive bacteria the proper interpretation of DNAase

128 resistance is less clear and has been equated with "uptake" or "internalization"

129 (37-39) without precise definition, although often assumed to indicate transport

130 to the cytoplasm. The latter assumption was reasonably suggested by the

131 observations that ComFA and ComEC, both required for the acquisition of DNAase

132 resistance, are almost certainly needed for transport to the cytoplasm, based on

133 their membrane localizations and molecular properties.

134 This study elucidates the process of tDNA uptake into the periplasm of $B$.

135 subtilis. We show here that DNAase resistance actually reflects entry of tDNA to

136 the periplasm, a step that we refer to as "uptake" to distinguish it from "transport"

137 across the cell membrane. With this understanding, we have visualized the

138 process of uptake using fluorescently labeled tDNA, enabling a re-evaluation of

139 the roles of several transformation proteins. Based on our results we present an

140 updated understanding of the early steps in transformation of B. subtilis, which

141 may be applicable to other transformable firmicutes.

\section{RESULTS}

144 Experimental design. To visualize uptake, bacteriophage lambda DNA (48.5 $145 \mathrm{kbp}$ ) was labeled using the Label-IT Rhodamine TM reagent (Mirus Bio) that 146 covalently couples the fluorophore predominantly to the N7-position of guanine 147 through a flexible linker, without disrupting Watson-Crick base pairing. To 148 minimize perturbation of the rhodamine-DNA (rDNA) structure we used a 149 labeling density that modifies fewer than $1 \%$ of the bases.

150 In B. subtilis, competence for transformation is expressed in only $10-20 \%$ of 151 the cells. To identify competent cells, the promoter of $\operatorname{com} G$ was fused at the amyE 152 locus to either yellow fluorescent protein (YFP) or cyan fluorescent protein (CFP), 153 which delineate the cytosolic compartments of the competent cells. These 154 isogenic strains behaved identically in our assays. For each experiment, aliquots 155 of YFP- and CFP-expressing competent cells were incubated separately with 156 rDNA, fixed with paraformaldehyde to stop transformation and then combined 
157 and visualized for epifluorescence and phase contrast microscopy. Depending on

158 the experiment, the YFP- and CFP-expressing samples were derived from wild-

159 type and mutant strains or from samples incubated with and without DNAase

160 after transformation. This permitted direct comparisons without differences due

161 to image processing and the vagaries of agarose pads.

162 DNAase as a tool for transformation studies. Studies in B. subtilis and $S$. 163 pneumoniae have used $50-200 \mu \mathrm{g} / \mathrm{ml}$ of DNAase under varying conditions to 164 investigate transformation $(4,19,34,37,38,40)$. Because we wished to study 165 uptake, we sought to standardize a DNAase treatment that would remove 166 attached rDNA from the surface of the cells, without degrading rDNA in the 167 unidentified protected compartment. Preliminary experiments comparing 168 treatment with DNAase concentrations from 10 to $100 \mu \mathrm{g} / \mathrm{ml}$ after 15 minutes of 169 incubation with rDNA, when the acquisition of DNAase resistance has reached 170 about half its maximum level (37), showed no differences in the intensities of the 171 rhodamine signals after DNAase treatment, comparing the lowest and highest 172 amounts of DNAase. If DNAase significantly penetrated the protected 173 compartment, we would have expected to see a dose-dependent decrease in the 174 residual rDNA signal. We therefore settled on a 3-minute incubation with 100 $175 \mu \mathrm{g} / \mathrm{ml}$ of DNAase at $37^{\circ} \mathrm{C}$ as an appropriate method for our experiments.

176 Wild-type cultures expressing YFP or CFP were each incubated with $0.2 \mu \mathrm{g} / \mathrm{ml}$ 177 rDNA, close to a saturation concentration for transformation. The CFP samples 178 were treated with DNAase and were then mixed with untreated YFP cells from the 179 same time points and visualized for fluorescence in the YFP, CFP and rhodamine 180 channels as well as by phase contrast after 2- and 30-minutes incubation with 181 rDNA. In the 2-minute sample (Figure 1A), few competent cells and very few of 182 the non-competent cells were associated with rDNA and after DNAase treatment 183 virtually no cells retained a significant rhodamine signal. After 30-minutes 184 incubation (Figure 1B), most of the competent cells showed an rDNA signal 185 without DNAase treatment while the non-competent cells were rarely $(<1 \%)$ 186 associated with rDNA, showing that association is biologically relevant. After 
187 DNAase treatment, many of the cells from the 30-minute sample were still

188 associated with rDNA (Figure 1B). The data presented to this point, are consistent

189 with a process in which DNA first binds specifically to the surface of competent 190 cells in a DNAase sensitive fashion and later becomes DNAase resistant by

191 entering a protected compartment. DNAase resistance is largely complete at 30

192 minutes, consistent with previous observations using radiolabeled tDNA (37),

193 suggesting that the uptake of rDNA is not markedly impeded by its covalent 194 adducts.

195 rDNA enters the periplasm where it becomes resistant to added DNAase.

196 It is likely that rDNA cannot cross the cell membrane because of its bulky adducts

197 (rhodamine plus a positively charged linker) and therefore accumulates in the 198 periplasm. Although we have found that rDNA with a labeling density similar to 199 that used in this study is reduced in its ability to transform, two considerations 200 make it impossible to interpret this reduced transforming capacity. First, low 201 density random labeling will leave variable stretches of label-free DNA that may 202 be capable of transforming. On the other hand, transformation could be blocked 203 by the adducts at the levels of recombination, gene expression or replication.

204 To investigate the location of DNAase-resistant rDNA further, we generated 205 3D-deconvolved, volume-reconstructed images of transformed CFP- and YFP206 labeled competent cells with and without DNAase treatment (Figure 1C and D).

207 The images show representative cells, viewed from 4 directions, related by $90^{\circ}$ 208 rotations around the long axis of the cell. The three images in Figure $1 \mathrm{C}$ show a 209 typical cell not treated with DNAase and imaged after 2 minutes of incubation with 210 rDNA, a time when very little tDNA is protected from DNAase. The projected views 211 show a blob of rDNA, exhibiting limited contact with the surface of the YFP 212 volume. $\lambda$ DNA molecules in solution are coils with an average radius of gyration 213 equal to about $500 \mathrm{~nm}$ (41). Figure $1 \mathrm{C}$ is consistent with binding of such an rDNA 214 coil to the cell surface, involving a limited portion of the rDNA surface. In cells 215 imaged after 30 minutes of incubation, whether DNAase-treated or not, the rDNA 216 is more closely juxtaposed to the surface (Figure 1D). In Figure 1D some of the 217 rhodamine signal is stretched across the surface, and comparison of the rotated 
218 views shows rDNA that appears to wrap around the cell volume, consistent with

219 its location within the confined space of the periplasm and its extension across the

220 outer face of the cell membrane. Figure 1E shows a single optical slice through

221 three cells, imaged after DNAase treatment following 30-minutes incubation with

222 rDNA, confirming that the rDNA signal is localized near the edges of the cells,

223 consistent with a periplasmic location and with the failure of rDNA to cross the

224 membrane. Figure 1C-E thus suggests a process in which rDNA initially associates

225 with the wall surface by localized contact and then enters the periplasmic

226 compartment where it becomes DNAase-resistant, while some of it then extends

227 across the cell membrane surface.

228 ComFA stabilizes ComGA. Before studying the roles of individual proteins in 229 rDNA uptake, it was important to determine the impact of each deletion mutation 230 on the stabilities of other proteins needed for transformation. Figure S1 shows 231 Western blots in which we have examined $\triangle \operatorname{comFA}, \triangle \operatorname{comGA}, \triangle$ nucA, $\triangle \operatorname{comC}$, $232 \triangle$ comEA and comEC518 loss-of-function mutant extracts using antisera raised 233 against ComGA, ComFA, NucA, ComEA and ComEC. All the mutations are deletions 234 except that comEC518 is a Tn917 insertion, which exhibits no transformability 235 and is located near the start of the comEC coding sequence after residue V50. All 236 of the proteins are present at close to normal levels in the mutants, with the 237 important exception of ComGA, which is destabilized in the absence of ComFA 238 (Figure S1B), as reported previously (42) and suggesting that ComGA and ComFA 239 are binding partners. However, ComGA is present at the normal level in an 240 inactivating K152E mutant of $\operatorname{comFA}(33,35)$. K152 lies in the Walker A motif of 241 ComFA, demonstrating that the ATPase activity of ComFA is not needed for 242 ComGA stability. In fact, ComGA interacts with both ComFA and ComFC (43). Thus, 243 any uptake-associated $\triangle$ comFA phenotype may be ascribed to an indirect effect on 244 ComGA

245 Testing mutants for rDNA binding and uptake. We next examined mixtures 246 of YFP- and CFP-labeled wild-type and mutant strains. To analyze the raw images, 247 we utilized several tools. First, we counted the percent of competent cells for 248 mutant and wild-type strains that were associated with detectable rDNA signals. 
249 Second, when required, we recorded deconvolved images and performed volume 250 reconstructions. These separate types of measurement were helpful because not 251 all the competent cells were associated with rDNA, even in the wild-type and 252 because in theory a given mutant might not have $100 \%$ penetrance, so that a few 253 cells might exhibit relatively normal binding or uptake while others are totally 254 deficient. It is important to recognize that the images recorded without DNAase 255 treatment reflect rDNA binding plus uptake, whereas after treatment they reflect 256 uptake to the periplasm.

257 ComGA and ComC. The $\triangle \operatorname{comGA}$ and $\triangle$ comC mutants exhibited no detectable 258 association with rDNA even without DNAase treatment (Figure S2). Because 259 ComGA is needed to assemble tpili and ComC is a peptidase that processes the 260 major and minor pilins for assembly (23), these results support the hypothesis 261 that tDNA binding requires tpili, most likely because the DNA binds directly to 262 these organelles, as it does in S. pneumoniae (20) and in V. cholerae (6).

263 ComFA. In the $\triangle \operatorname{comFA}$ strain, there is a significant decrease in the percent of 264 cells that were associated with rDNA after 30-minutes, either with or without 265 DNAase treatment (Table 1). Despite this, many $\triangle c o m F A$ cells did bind and take 266 up rDNA. In the absence of DNAase treatment the signal intensities of the wild267 type and mutant cells were similar (Figure S3, panel A) but with treatment the 268 signals in the mutant were markedly weaker (Figure S3, panel B), in agreement 269 with results obtained using radiolabeled tDNA $(32,34)$. If the decrease in signal 270 intensity in the $\triangle c o m F A$ strain is due to a deficiency in ComGA (Figure S1, panel 271 B), we would expect the comFA K152E mutant to behave like the wild-type. 272 Indeed, no significant effect of the K152E mutant was observed on the percent of 273 cells showing rDNA fluorescence either with or without DNAase treatment (Table 274 1) and the images in Figure 2A and 2B show that there are no obvious effects of 275 the mutation on either the total or DNAase resistant signal intensities. We 276 conclude that the effect of $\triangle c o m F A$ on the acquisition of DNAase resistance and on 277 rDNA binding to the cells is largely due to ComGA deficiency. Despite the non278 requirement of ComFA for uptake, the rDNA signal in the K152E mutant cells is 279 more localized after DNAase treatment than in the wild-type cells, suggesting an 
280 unexpected ATP-dependent requirement for ComFA in the spreading of the rDNA 281 in the periplasm (Figure 2C).

282 ComEA. In a $\triangle$ comEA strain the percent of cells with associated rDNA signals 283 was reduced about 7-fold in the absence of DNAase treatment (Figure 3A and 284 Table 1) and the frequency of cells with DNAase resistant rDNA was reduced 57285 fold (Figure 3B and Table 1), consistent with results obtained with B. subtilis and 286 S. pneumoniae using radiolabeled DNA $(19,37,38)$. In the $\triangle$ comEA cells that did 287 exhibit binding, the rDNA remained on the cell surface (Figure 3C), like the wild288 type image at 2-minutes (Figure 1A). Thus, ComEA assists in binding and is 289 required for uptake. Most likely, the initial binding of tDNA to the tpilus is labile 290 and must be stabilized by contact with periplasmic ComEA, a known DNA-binding 291 protein. Indeed, reversible attachment of DNA to the surface of $B$. subtilis 292 competent cells has been reported (44). The nearly total DNAase susceptibility of 293 rDNA in the mutant demonstrates that ComEA is also needed for uptake to the 294 periplasm.

295 NucA. A $\triangle n u c A$ strain exhibits decreased rates of transformation, tDNA 296 binding and the acquisition of DNAase resistance (37). To explore this phenotype 297 with rDNA, we used samples incubated with rDNA for 15-rather than 30-minutes 298 to maximize the differences between the mutant and wild-type strains, because 299 the final yield of transformants in the mutant is only reduced about 2-fold (37). 300 The percent of $\triangle n u c A$ cells associated with rDNA (Table 1) was reduced about 2301 fold and the rDNA signal intensities were clearly reduced after DNAase treatment 302 (Figure S4). To determine if the uptake deficiency of the $\Delta n u c A$ strain was due to 303 the nuclease activity of NucA, we inserted a D98A active site mutation at the native 304 nuсA locus. The design of this mutation was based on analysis of NucB (45), which 305 exhibits 58\% identity with NucA. D98A corresponds in position to residue D87A 306 in NucB. This mutant exhibits a transformation deficiency similar to that of the $307 \Delta n u c A$ strain. The data in Table 1 show that the D98A strain has a similar 308 phenotype to the knockout of $n u c A$, although the effect on the frequency of cells 309 associated with rDNA is not as great, perhaps due to residual nuclease activity. 310 Figure 4 shows that the intensities of the rDNA signals in this mutant were 
311 decreased following DNAase treatment, confirming that the nuclease activity of

312 NucA is needed for uptake to the periplasm and excluding a polar effect of nucA

313 inactivation on the downstream nin gene (46). Volume reconstructions after

314 DNAase treatment for the $\triangle n u c A$ (Figure S5 A) and D98A (Figure S5 B) mutants

315 show that the rDNA is more localized than in the wild-type. similar to the

316 phenotypes of the comFA K152E and comEC518 mutants (see below). Based on

317 the earlier assumption that DNAase resistance corresponds to entry to the

318 cytoplasm, it was proposed that NucA produced ends for transport through the

319 membrane channel (37). Although this may be true, the present results show that

320 the nuclease activity also plays a role in uptake to the periplasm.

321 ComEC. As noted above, the use of radiolabeled rDNA showed that the

322 elimination of ComEC did not affect the total association of tDNA with competent

323 cells but markedly reduced the label after DNAase treatment $(4,37,38)$. The use

324 of rDNA should therefore show a previously unexpected role for ComEC in uptake.

325 To test this, we used the comEC518 mutation, which has a transposon insertion

326 near the N-terminus of the comEC coding sequence (47). After 30 minutes, the

327 fraction of competent cells showing DNAase-resistant rDNA was moderately

328 decreased in the comEC518 mutant (31\%) compared with 67\% for the wild-type

329 (Table 1), probably a significant difference $(\mathrm{p}=0.01)$. In contrast, there is little if

330 any effect on the frequency of rDNA attachment before DNAase treatment. This

331 trend is confirmed by the images contained in Figure 5. Panel A shows that in the

332 absence of DNAase treatment, the intensities of the rDNA signals are comparable

333 in wild-type and mutant cells. However, Figure 5B shows that the rDNA signal in

334 those DNAase-treated comEC518 cells that exhibit uptake is markedly lower than

335 in the wild-type, in agreement with measurements made using radiolabeled tDNA

$336(4,19,34,37)$. Interestingly, the volume reconstructed images in Figure $5 \mathrm{C}$ reveal

337 a marked difference between the DNAase-resistant rDNA signals in wild-type and

338 comEC cells. In the wild-type cells, as noted above (Figure 1B), some of the rDNA

339 is extended across the volume surface. But in the comEC518 cells, the DNAase

340 resistant rDNA is more localized. Thus, although binding to the cell surface is 
341 essentially normal in the comEC mutant, uptake is strongly affected and the 342 reduced amount of rDNA that manages to enter the periplasm is confined locally.

343 rDNA closely associates with YFP-ComEA during uptake. In the Gram344 negative systems, periplasmic ComEA is initially distributed uniformly within the 345 periplasm and then dramatically forms foci, accumulating at the sites of DNA 346 uptake $(8,9)$. To examine the interactions of rDNA with ComEA in vivo, we 347 constructed a YFP-ComEA mutant in which the N-terminal YFP moiety was in the 348 cytosol and the DNA binding portion of ComEA was appropriately located in the 349 periplasm. The $y f p$-comEA construct was placed at the comE locus, expressed 350 normally under competence control. The resulting strain, with $y f p$-comEA as the 351 only source of $\operatorname{com} E A$ in the cell, was normally transformable, although the rate of 352 appearance of DNAase resistant transformants was deceased about 2-fold. As 353 reported previously using immunofluorescence with native protein (48) and by 354 Kaufenstein et al (49) with a YFP-ComEA construct similar to ours, ComEA forms 355 large foci at several locations around the membrane. Uniquely among the 356 transformation proteins there is no obvious preference of these foci for polar or 357 sub-polar locations. The foci are not caused by the uptake of DNA from occasional 358 lysed cells, because the addition of DNAase $(100 \mu \mathrm{g} / \mathrm{ml})$ during 90 minutes of 359 growth prior to sampling had no effect on this distribution.

360 After the addition of rDNA, the distribution of YFP-ComEA foci was not 361 obviously different in the cells with and without bound rDNA. Figure 6A shows 362 deconvolved and volume reconstructed images of four representative cells from 363 a single field that exhibit significant rDNA attachment after I minute incubation 364 with rDNA. In these images, the rhodamine signal is almost always localized near 365 the poles as reported previously using a different DNA-labeling protocol (48). The 366 rDNA in Figure 6A, is consistently near a blob of YFP-ComEA in all the cells shown 367 except in panel Aa, which may show a cell with initial attachment to a tpilus that 368 is not located near an accumulation of ComEA. In the remaining cells of panel A, 369 the rDNA and YFP-ComEA appear to be connected by faint regions of YFP signal 370 intensity. After 10 minutes of incubation the images are strikingly different 371 (Figure 6B). At this time, the rDNA is often intimately associated with foci of YFP- 
372 ComEA and is sometimes located away from the cell poles (Figure 6B, cells c and

373 d). At this time, in this same experiment, much of the rDNA intensity is resistant

374 to DNAase. These images are consistent with a scenario in which tDNA initially

375 attaches to tpili at the poles, and is then pulled into the periplasm, where it binds

376 to locally mobile molecules of ComEA. The Graumann laboratory has reported

377 that the diffusive behavior of YFP-ComEA in the membrane is similar to that of

378 tDNA in the periplasm, suggesting that these molecules are associated (49),

379 consistent with this conclusion. After the capture event, which results in stabilized

380 binding but no major redistribution of ComEA, the tDNA is plausibly pulled into

381 the periplasm by a Brownian ratchet mechanism involving ComEA and ComEC,

382 where it is stretched across clusters of ComEA molecules.

\section{DISCUSSION}

385 The new appreciation of the meaning of DNAase resistance allows us to 386 interpret data acquired in this study and to reinterpret data from the literature. It 387 had been generally assumed that the acquisition of resistance was due to 388 transport of tDNA across the cell membrane, but our present results show that 389 resistance is actually imparted by the cell wall barrier. DNAase, has a calculated 390 molecular weight of $29 \mathrm{kDa}$ and it has been estimated from in vitro studies that 391 globular proteins of more than about $25 \mathrm{kDa}$ cannot diffuse through the cell wall 392 of $B$. subtilis (50). The wall preparations used to produce this estimate were 393 treated to remove associated proteins and the intact wall is likely to be even 394 denser and thus more restrictive to diffusion.

395 The conceptual partition of transformation proteins into three exclusive 396 categories based on their requirements for binding, uptake and transport, does 397 not fit with the new data. Thus, ComEA is needed for both tight binding and 398 uptake. ComGA appears to be needed for both initial binding and for uptake 399 because the $\triangle c o m F A$ mutation, which lowers the amount of ComGA, decreases the 400 frequency of rDNA binding (Table 1) and is deficient in the amount of uptake in 401 individual cells (Figure S3), while the comFA K152E mutation does not reduce 
402 uptake. NucA, ComEC and ComFA help to distribute tDNA within the periplasm

403 and the latter two proteins are certainly also needed for transport to the 404 cytoplasm, while NucA helps with binding as well as uptake. This complexity is 405 consistent with the participation of the proteins in a nanomachine, in which they 406 interact to guide tDNA through the wall, periplasm and membrane. Further 407 support for this notion is provided by the observations that ComFA stabilizes 408 ComGA (Fig, S1) and that ComFC binds to ComFA (31). Co-immunoprecipitation 409 experiments in B. subtilis have shown that ComGA associates with ComFA, ComFC, 410 DprA, SsbB, RecA and ComEC (43), all proteins that participate in transformation, 411 providing support for the notion of a nanomachine.

412 The results for $\triangle$ comGA and $\triangle \operatorname{comC}$ conform to previous data for B. subtilis and 413 S. pneumoniae obtained using radiolabeled DNA $(24,44,51)$ showing that in the 414 absence of these proteins there is no detectable binding of tDNA to the mutant 415 cells. This reinforces the belief that the tpilus is the initial site of tDNA binding and 416 is consistent with the localization of both ComGA and the initial sites of DNA 417 binding near the poles (48). tpilus retraction has now been observed in both $V$. 418 cholerae and S. pneumoniae $(6,22)$, and is very likely to also occur in $B$. subtilis, 419 although both of these Gram-positive organisms lack retraction ATPases. Based 420 on a number of recent studies it is tempting to conclude that the $B$. subtilis pilus 421 retracts by spontaneous disassembly into the membrane without an external 422 energy source $(6,52-54)$. However, a dual function ATPase has been described 423 that drives both assembly and retraction of a t4 pilus (55), and this remains a 424 possibility for ComGA. In fact, the major effect of $\triangle$ comFA on uptake compared to 425 binding (Table 1, Figure S3), could be explained by the decreased amount of 426 ComGA (Figure S1),

427 In the absence of ComEA, tDNA binding is reduced, and uptake is eliminated. 428 There is experimental evidence for an initially transient form of binding (44), 429 suggesting that attachment of tDNA to the tpilus is reversible. ComEA is too small 430 to extend from the membrane through the wall to aid in the initial binding, and 431 attachment of tDNA to the cell is likely to be stabilized by contact between the 432 tDNA and ComEA in the periplasm. During the uptake of tDNA, no dramatic 
433 relocation of the membrane-anchored YFP-ComEA comparable to the

434 reorganization reported for Vibrio and Neisseria $(8,9,12)$ was observed. We

435 propose that inwardly diffusing DNA segments are first captured by fixed or

436 locally mobile ComEA molecules. Further uptake and the spreading of rDNA

437 within the periplasm then results from the inward diffusion of additional

438 segments of DNA, which are trapped by more distant ComEA molecules, in an on-

439 going Brownian ratchet process. The dependence of uptake on NucA and ComEC

440 and the restricted distribution of DNAase resistant rDNA in the comEC518, nucA

441 and comFA K152E mutants suggests that a more complex mechanism takes place

442 than in the Gram-negatives, where uptake depends only on the tpilus and ComEA.

443 This complexity may be characteristic of bacteria with membrane-anchored, 444 relatively immobile ComEA.

445 A role for ComEC in uptake to the periplasm was unexpected. The B. subtilis

446 ComEC contains two conserved periplasmic domains that are obvious candidates

447 for involvement in uptake $(29,56,57)$ : the N-terminal N-loop or OB-domain

448 (PFAM13567) and the C-terminal metallo- $\beta$-lactamase domain (PFAM00753)

449 (57). Between the two is the competence domain (PFAM03772) that contains

450 several transmembrane helices and must contribute to forming the transport

451 channel. The restricted localization of rDNA in comEC and comFA mutants suggest

452 that tDNA is transferred to ComEC in a process that depends on ComFA. The

453 absence of ComEC might then arrest uptake by confining the rDNA to locally

454 available ComEA. Transfer to ComEC would also position tDNA for entry to the

455 channel and for degradation of the non-transforming strand. A recent finding in

456 Helicobacter pylori (58) is consistent with these ideas. This organism encodes

457 neither tpili nor ComEA but uses a type 4 secretion system to bring tDNA into the

458 periplasm and ComH, a periplasmic DNA binding protein that interacts with tDNA

459 in the periplasm. Suggestively, ComH, which does not resemble ComEA but plays

460 an analogous role, interacts directly with the OB-domain of ComEC (58). It has

461 been proposed that the $\beta$-lactamase domain contains the nuclease activity that

462 degrades the non-transforming strand of tDNA (57). If degradation does take 
463 place in the periplasm, ComEC may provide an entropic boost to uptake by 464 converting tDNA to single strands and free nucleotides.

465 The role of NucA in uptake is also a novel finding. NucA is anchored in the 466 membrane with its catalytic site protruding into the periplasm. Its nuclease 467 activity assists in uptake (Table 1, Figure 4) implying that either a nick on one 468 strand of tDNA or a double strand cut facilitate uptake. Indeed, a double strand 469 cut is likely, as suggested by studies of the NucA paralog, NucB (57). 470 Transformation with circular DNA has shown NucA-dependent conversion to the 471 full length linear form shortly after binding (37). This result reinforces the idea 472 that tDNA crosses the wall as a folded dsDNA molecule. Folding DNA, which has a 473 persistence length of about $50 \mathrm{~nm}(\sim 147 \mathrm{bp})$, requires local deformation of the 474 DNA structure, presumably caused by binding to the tpilus. After release from the 475 tpilus, strain in the folded tDNA would impel the tDNA to straighten locally and 476 pull back through the wall. A double strand break induced by NucA would relieve 477 the strain and accelerate the ratchet. An additional explanation for the role of 478 NucA, which is not mutually exclusive with this one, is suggested by the finding 479 that the ComEA protein of $V$. cholerae prefers binding to a DNA end (8). If this is 480 also true of the $B$. subtilis protein, NucA might not only relieve the strain in folded 481 tDNA but would also facilitate the initial binding of ComEA to the incoming DNA. 482 These ideas are consistent with our finding that both binding and uptake are 483 reduced in the $n u c A$ mutants (Table 1, Figure 4). The termini produced by NucA 484 would also be available to enter the ComEC channel (37).

485 The results presented in this study allow us to propose an updated model for 486 transformation in B. subtilis. First, tDNA binds reversibly to the tpilus, which 487 retracts, bringing a folded loop of DNA into the periplasm. The $S$. pneumoniae 488 tpilus is $6 \mathrm{~nm}$ thick and retraction would likely leave a hole in the wall of the same 489 size. A folded DNA molecule would have thickness of $4 \mathrm{~nm}$, permitting the tpilus 490 to bring the DNA into the periplasm with its hydration shell intact, and allowing 491 subsequent inward diffusion of the DNA. NucA then cuts the tDNA as it is released 492 from the tpilus, relieving strain introduced by tDNA folding and possibly 493 facilitating binding to ComEA at a terminus. This binding stabilizes the association 
494 of tDNA with the cell and as successive segments of DNA diffuse into the periplasm

495 through the cell wall, they are captured by additional ComEA molecules and 496 prevented from diffusing outward. As the ComEA binding sites near the locus of 497 entry become occupied, tDNA spreads in a process dependent on ComFA and 498 ComEC and is captured by ComEC for transport to the cytoplasm, accomplished 499 by ComEC and the ComFA/ComFC complex, which comprise the transformation 500 permease.

501 The use of labeled DNA that cannot cross the membrane may obscure what is 502 actually a seamless process of uptake and transport. In other words, 503 transformation may not be a two-step process in which tDNA accumulates in the 504 periplasm. Another open question concerns the extent to which our revised 505 understanding of DNAase resistance applies to other Gram-positive 506 transformable bacteria, such as S. pneumoniae. Despite the shared requirements 507 for ComEA, ComEC and $\mathrm{t} 4$ pilus proteins, there are likely to be important 508 mechanistic differences between the uptake mechanisms of these two model 509 Gram-positive bacteria. In S. pneumoniae, degradation of the non-transforming 510 strand is accomplished by EndA $(59,60)$ and degradation takes place in the 511 absence of ComEC (38), implying that the latter is not needed for uptake to the 512 periplasm. In contrast, B. subtilis does not encode EndA and when comEC is 513 inactivated, degradation ceases (37), consistent with the role of ComEC in uptake 514 and perhaps with its proposed action as a nuclease. Finally, in B. subtilis the tpilus 515 is probably short while in S. pneumoniae it extends from the cell surface $(20,23)$. 516 Many outstanding questions remain, most importantly, how these various 517 processes are accomplished on the molecular level.

\section{Materials and Methods}

520 Strains and growth conditions. All mutant strains (Table S1) of B. subtilis

521 were constructed in IS75 (his leu met) and are derivatives of the domesticated 522 strain 168. All the strains expressed YFP or CFP controlled by the comG 523 promoter, inserted ectopically in amyE. B. subtilis was grown to competence for 
524 all experiments by the two-step method (61) except that unfrozen cultures were

525 used and cultures were started from plates grown overnight instead of from

526 spore suspensions.

527 Transformation for microscopy. Competent cultures of $\mathrm{P}_{\mathrm{G}}-\mathrm{CFP}$ and $\mathrm{P}_{\mathrm{G}}-\mathrm{YFP}$

528 expressing mutant and wild-type strains were mixed 1:1 and the mixture was

529 incubated with $0.2 \mu \mathrm{g} / \mathrm{ml}$ of rhodamine-labeled DNA for the indicated times, in

530 final volumes of 300-500 $\mu$ l. Samples were taken and either immediately fixed by

531 the addition of 3.2\% paraformaldehyde and incubation at room temperature for

53230 minutes or were first incubated for 3 minutes with $100 \mu \mathrm{g} / \mathrm{ml}$ DNAase I at 37o

$533 \mathrm{C}$ before fixation. In the experiment shown in Figure 1, where DNAase-treated

534 and untreated samples were combined for visualization, the cells were

535 centrifuged briefly and resuspended in the same medium used to achieve

536 competence, but containing no $\mathrm{Mg}$ and EDTA (10 $\mathrm{mM})$ to stop the nuclease

537 activity before mixing and fixation. Samples were then washed and imaged.

538 Preparation of rDNA. Bacteriophage $\lambda$ DNA (New England Biolabs) was

539 diluted to $10 \mu \mathrm{g} / \mathrm{ml}$ and incubated for 60 minutes with a MIRUS Label-IT

540 rhodamine TM reagent (Mirus Bio) according to the manufacturer's

541 recommendations. The DNA was then processed through a spin column to

542 remove excess reagent.

543 Point mutation of nucA. A fragment of DNA carrying the nucA sequence

544 with a D98A mutation and BamH1 ends, was synthesized by Biomatik (Canada)

545 and cloned into the BamH1 site of pMiniMAD2, a gift from Dan Kearns (Indiana

546 University). After transformation with selection for erythromycin $(5 \mu \mathrm{g} / \mathrm{ml})$, the

547 markerless chromosomal mutant was isolated as described by Patrick and

548 Kearns (62).

549 YFP-ComEA construction. Three fragments were produced by PCR and

550 assembled using NEBuilder HiFi into pUC18CM, a derivative of pUC18 with a

551 chloramphenicol resistance cassette for selection in B. subtilis.. Fragment \#1

552 carried 1500 base pairs upstream from the comEA coding sequence including the

553 promoter, the ribosomal binding site and start codon. To produce this fragment

554 primers 1 and 2 were used (Table S2). Fragment \#2 carried the $y f p$ open reading 
555 frame with the stop codon omitted, produced using primers 3 and 4. Fragment

556 \#3 consisted of the comEA open reading frame without its start codon, produced

557 using primers 5 and 6. After assembly, the clone was verified by sequencing and

558 transformed into the native locus via a single crossover event. The duplicated

559 native comEA open reading frame was then deleted by transformation using

560 DNA from strain BD8739 (comEA::ery trpC2) and the final construction was

561 again verified by sequencing.

562 Microscopy. For microscopy $1 \mu$ samples of transformed cells were placed on

563 thin agarose pads before visualization. All images were acquired with a 100x Plan

564 Appo immersion objective, NA=1.40 on a Nikon Ti microscope, equipped with LED

565 excitation sources and an Orca Flash 4.0 camera (Hamamatsu). Nikon Elements

566 was used for data acquisition and image analysis. Z-stack images were recorded

567 with $200 \mathrm{~nm}$ steps and processed for 3D deconvolution with the Landweber

568 algorithm in Nikon Elements. Volume reconstructions were assembled with alpha

569 blending. Images were exported to Adobe Photoshop and Microsoft PowerPoint

570 for final processing.

571 Western blotting. Western blotting was carried out using standard methods

572 with semi-dry blotting and the nitrocellulose blots were developed using ECL (GE

573 Healthcare). The images were recorded with a BioRad ChemiDoc MP imager. In

574 most gels, for a loading control, the top of the membrane was cut off after blotting

575 and developed separately with anti-EFG antiserum, a kind gift from Jonathan

576 Dworkin (Columbia University Medical school).

577 Statistical analysis. Proportions of mutant and wild-type cells that were 578 associated with rDNA were compared using a two-tailed z-test.

579 Data availability statement. The data that support the findings of this study

580 are available from the corresponding author, [author initials], upon reasonable

581 request.

582

\section{SUPPLEMENTAL MATERIAL}

584 Table S1 Strains 


\section{Table S2 Primers}

586

587 Figure S1. Western blots for transformation proteins in mutant strains.

588 Figure S2. DNA binding in $\triangle \operatorname{com} G A$ and $\triangle \operatorname{comC}$ strains.

589 Figure S3. Binding and uptake in the $\triangle$ comFA mutant.

590 Figure S4. Binding and uptake in the $\Delta n u c A$ mutant.

591 Figure S5. Volume reconstructions of the $\triangle n u c A$ and nucA D98A mutant strains.

592

\section{ACKNOWLEDGEMENTS}

594 This work was supported by NIH grant R01GM057720. We thank Mathew

595 Neiditch and members of the Dubnau lab for helpful comments and discussion.

596 We thank Daniel Ziegler at the Bacillus Genetic Stock Center for providing

597 strains and J. Dworkin for the anti-EFG antiserum. J. H. and M. D. S. performed all

598 of the experimental work. D. D. supervised the work and all the authors

599 contributed to planning experiments, analyzing data and to writing the paper. 


\section{REFERENCES}

602

603 1. Blokesch M. 2016. Natural competence for transformation. Curr Biol 604 26:R1126-R1130.

605 2. Dubnau D, Blokesch M. 2019. Mechanisms of DNA Uptake by Naturally 606 Competent Bacteria. Annu Rev Genet 53:217-237.

607 3. Maier B. 2020. Competence and Transformation in Bacillus subtilis. Curr $608 \quad$ Issues Mol Biol 37:57-76.

609

4. Hahn J, Albano M, Dubnau D. 1987. Isolation and characterization of

610

611

612

613

614

615

616

617

618

619

620

621

622

623

624

625

626

627

628

629

630

631

632

633

634

635

636

637

638

639

640

641

642

643 Tn917lac-generated competence mutants of Bacillus subtilis. J Bacteriol 169:3104-9.

5. Albano M, Hahn J, Dubnau D. 1987. Expression of competence genes in Bacillus subtilis. J Bacteriol 169:3110-7.

6. Ellison CK, Dalia TN, Vidal Ceballos A, Wang JC, Biais N, Brun YV, Dalia AB. 2018. Retraction of DNA-bound type IV competence pili initiates DNA uptake during natural transformation in Vibrio cholerae. Nat Microbiol 3:773-780.

7. Seitz P, Blokesch M. 2014. DNA transport across the outer and inner membranes of naturally transformable Vibrio cholerae is spatially but not temporally coupled. MBio 5.

8. Seitz P, Pezeshgi Modarres H, Borgeaud S, Bulushev RD, Steinbock LJ, Radenovic A, Dal Peraro M, Blokesch M. 2014. ComEA is essential for the transfer of external DNA into the periplasm in naturally transformable Vibrio cholerae cells. PLoS Genet 10:e1004066.

9. Gangel H, Hepp C, Muller S, Oldewurtel ER, Aas FE, Koomey M, Maier B. 2014. Concerted spatio-temporal dynamics of imported DNA and ComE DNA uptake protein during gonococcal transformation. PLoS Pathog 10:e1004043.

10. Peskin CS, Odell GM, Oster GF. 1993. Cellular motions and thermal fluctuations: the Brownian ratchet. Biophys J 65:316-24.

11. Stingl K, Muller S, Scheidgen-Kleyboldt G, Clausen M, Maier B. 2010. Composite system mediates two-step DNA uptake into Helicobacter pylori. Proc Natl Acad Sci U S A 107:1184-9.

12. Hepp C, Maier B. 2016. Kinetics of DNA uptake during transformation provide evidence for a translocation ratchet mechanism. Proc Natl Acad Sci U S A 113:12467-12472.

13. Matias VR, Beveridge TJ. 2005. Cryo-electron microscopy reveals native polymeric cell wall structure in Bacillus subtilis 168 and the existence of a periplasmic space. Mol Microbiol 56:240-51.

14. Matias VR, Beveridge TJ. 2008. Lipoteichoic acid is a major component of the Bacillus subtilis periplasm. J Bacteriol 190:7414-8.

15. Zuber B, Haenni M, Ribeiro T, Minnig K, Lopes F, Moreillon P, Dubochet J. 2006. Granular layer in the periplasmic space of gram-positive bacteria and fine structures of Enterococcus gallinarum and Streptococcus gordonii 
645

646

647

648

649

650

651

652

653

654

655

656

657

658

659

660

661

662

663

664

665

666

667

668

669

670

671

672

673

674

675

676

677

678

679

680

681

682

683

684

685

686

687

688

689

690

septa revealed by cryo-electron microscopy of vitreous sections. J Bacteriol 188:6652-60.

16. Reichmann NT, Grundling A. 2011. Location, synthesis and function of glycolipids and polyglycerolphosphate lipoteichoic acid in Gram-positive bacteria of the phylum Firmicutes. FEMS Microbiol Lett 319:97-105.

17. Mirouze N, Ferret C, Cornilleau C, Carballido-Lopez R. 2018. Antibiotic sensitivity reveals that wall teichoic acids mediate DNA binding during competence in Bacillus subtilis. Nat Commun 9:5072.

18. Provvedi R, Dubnau D. 1999. ComEA is a DNA receptor for transformation of competent Bacillus subtilis. Mol Microbiol 31:271-80.

19. Inamine GS, Dubnau D. 1995. ComEA, a Bacillus subtilis integral membrane protein required for genetic transformation, is needed for both DNA binding and transport. J Bacteriol 177:3045-51.

20. Laurenceau R, Pehau-Arnaudet G, Baconnais S, Gault J, Malosse C, Dujeancourt A, Campo N, Chamot-Rooke J, Le Cam E, Claverys JP, Fronzes R. 2013. A type IV pilus mediates DNA binding during natural transformation in Streptococcus pneumoniae. PLoS Pathog 9:e1003473.

21. Sheppard D, Berry JL, Denise R, Rocha EPC, Matthews S, Pelicic V. 2020. The major subunit of widespread competence pili exhibits a novel and conserved type IV pilin fold. J Biol Chem 295:6594-6604.

22. Lam T, Ellison CK, Dalia AB, Eddington DT, Morrison DA. 2021. Type IV competence pili in Streptococcus pneumoniae are highly dynamic structures that retract to promote DNA uptake. bioRxiv doi:10.1101/2021.01.17.426607:2021.01.17.426607.

23. Chen I, Provvedi R, Dubnau D. 2006. A Macromolecular Complex Formed by a Pilin-like Protein in Competent Bacillus subtilis. J Biol Chem 281:21720-7.

24. Briley K, Jr., Dorsey-Oresto A, Prepiak P, Dias MJ, Mann JM, Dubnau D. 2011. The secretion ATPase ComGA is required for the binding and transport of transforming DNA. Mol Microbiol 81:818-30.

25. Chung YS, Dubnau D. 1995. ComC is required for the processing and translocation of ComGC, a pilin- like competence protein of Bacillus subtilis. Mol Microbiol 15:543-51.

26. Chung YS, Dubnau D. 1998. All seven comG open reading frames are required for DNA binding during transformation of competent Bacillus subtilis. J Bacteriol 180:41-5.

27. Meima R, Eschevins C, Fillinger S, Bolhuis A, Hamoen LW, Dorenbos R, Quax WJ, van Dijl JM, Provvedi R, Chen I, Dubnau D, Bron S. 2002. The $b d b D C$ operon of Bacillus subtilis encodes thiol-disulfide oxidoreductases required for competence development. J Biol Chem 277:6994-7001.

28. Mann JM, Carabetta VJ, Cristea IM, Dubnau D. 2013. Complex formation and processing of the minor transformation pilins of Bacillus subtilis. Mol Microbiol 90:1201-15.

29. Draskovic I, Dubnau D. 2005. Biogenesis of a putative channel protein, ComEC, required for DNA uptake: membrane topology, oligomerization and formation of disulphide bonds. Mol Microbiol 55:881-96. 
691 30. Chung YS, Breidt F, Dubnau D. 1998. Cell surface localization and

692

693

694

695

696

697

698

699

700

701

702

703

704

705

706

707

708

709

710

711

712

713

714

715

716

717

718

719

720

721

722

723

724

725

726

727

728

729

730

731

732

733

734

processing of the ComG proteins, required for DNA binding during transformation of Bacillus subtilis. Mol Microbiol 29:905-913.

31. Diallo A, Foster HR, Gromek KA, Perry TN, Dujeancourt A, Krasteva PV, Gubellini F, Falbel TG, Burton BM, Fronzes R. 2017. Bacterial transformation: ComFA is a DNA-dependent ATPase that forms complexes with ComFC and DprA. Mol Microbiol 105:741-754.

32. Londono-Vallejo JA, Dubnau D. 1993. comF, a Bacillus subtilis late competence locus, encodes a protein similar to ATP-dependent RNA/DNA helicases. Mol Microbiol 9:119-31.

33. Londono-Vallejo JA, Dubnau D. 1994. Mutation of the putative nucleotide binding site of the Bacillus subtilis membrane protein ComFA abolishes the uptake of DNA during transformation. J Bacteriol 176:4642-5.

34. Londono-Vallejo JA, Dubnau D. 1994. Membrane association and role in DNA uptake of the Bacillus subtilis PriA analogue ComF1. Mol Microbiol 13:197-205.

35. Chilton SS, Falbel TG, Hromada S, Burton BM. 2017. A Conserved Metal Binding Motif in the Bacillus subtilis Competence Protein ComFA Enhances Transformation. J Bacteriol 199.

36. Mejean V, Claverys JP. 1993. DNA processing during entry in transformation of Streptococcus pneumoniae. J Biol Chem 268:5594-9.

37. Provvedi R, Chen I, Dubnau D. 2001. NucA is required for DNA cleavage during transformation of Bacillus subtilis. Mol Microbiol 40:634-644.

38. Bergé M, Moscoso M, Prudhomme M, Martin B, Claverys JP. 2002. Uptake of transforming DNA in Gram-positive bacteria: a view from Streptococcus pneumoniae. Mol Microbiol 45:411-21.

39. Boonstra M, Vesel N, Kuipers OP. 2018. Fluorescently Labeled DNA Interacts with Competence and Recombination Proteins and Is Integrated and Expressed Following Natural Transformation of Bacillus subtilis. mBio 9.

40. Burghard-Schrod M, Kilb A, Krämer K, Graumann PL. 2020. Single molecule dynamics of DNA receptor ComEA, membrane permease ComEC and taken up DNA in competent Bacillus subtilis cells. bioRxiv doi:10.1101/2020.09.29.319830:2020.09.29.319830.

41. Phillips R, Kondev J, Theriot J, Garcia HG, Orme N. 2013. Physical Biology of the Cell. Garland Science, London and New York.

42. Kramer N, Hahn J, Dubnau D. 2007. Multiple interactions among the competence proteins of Bacillus subtilis. Mol Microbiol 65:454-64.

43. Hahn J, Tanner AW, Carabetta VJ, Cristea IM, Dubnau D. 2015. ComGA-RelA interaction and persistence in the Bacillus subtilis K-state. Mol Microbiol 97:454-71.

44. Maier B, Chen I, Dubnau D, Sheetz MP. 2004. DNA transport into Bacillus subtilis requires proton motive force to generate large molecular forces. Nat Struct Mol Biol 11:643-9. 
735 45. Basle A, Hewitt L, Koh A, Lamb HK, Thompson P, Burgess JG, Hall MJ,

736

737

738

739

740

741

742

743

744

745

746

747

748

749

750

751

752

753

754

755

756

757

758

759

760

761

762

763

764

765

766

767

768

769

770

771

772

773

774

775

776

777

778 Hawkins AR, Murray H, Lewis RJ. 2018. Crystal structure of NucB, a biofilmdegrading endonuclease. Nucleic Acids Res 46:473-484.

46. Vosman B, Kuiken G, Venema G. 1988. Transformation in Bacillus subtilis: Involvement of the 17-kilodalton DNA-entry nuclease and the competence-specific 18-kilodalton protein. J Bacteriol 170:3703-3710.

47. Hahn J, Inamine G, Kozlov Y, Dubnau D. 1993. Characterization of comE, a late competence operon of Bacillus subtilis required for the binding and uptake of transforming DNA. Mol Microbiol 10:99-111.

48. Hahn J, Maier B, Haijema BJ, Sheetz M, Dubnau D. 2005. Transformation proteins and DNA uptake localize to the cell poles in Bacillus subtilis. Cell 122:59-71.

49. Kaufenstein M, van der Laan M, Graumann PL. 2011. The three-layered DNA uptake machinery at the cell pole in competent Bacillus subtilis cells is a stable complex. J Bacteriol 193:1633-42.

50. Demchick P, Koch AL. 1996. The permeability of the wall fabric of Escherichia coli and Bacillus subtilis. J Bacteriol 178:768-73.

51. Albano M, Dubnau DA. 1989. Cloning and characterization of a cluster of linked Bacillus subtilis late competence mutations. J Bacteriol 171:5376-85.

52. Zollner R, Cronenberg T, Maier B. 2019. Motor properties of PilTindependent type 4 pilus retraction in gonococci. J Bacteriol doi:10.1128/JB.00778-18.

53. Clausen M, Jakovljevic V, Sogaard-Andersen L, Maier B. 2009. High-force generation is a conserved property of type IV pilus systems. J Bacteriol 191:4633-8.

54. Chlebek JL, Craig L, Dalia AB. 2021. Type IV pili share a conserved mechanism of motor-independent retraction that is an inherent property of the pilus filament. bioRxiv doi:10.1101/2021.02.10.430637:2021.02.10.430637.

55. Ellison CK, Kan J, Chlebek JL, Hummels KR, Panis G, Viollier PH, Biais N, Dalia AB, Brun YV. 2019. A bifunctional ATPase drives tad pilus extension and retraction. Sci Adv 5:eaay2591.

56. Pimentel ZT, Zhang Y. 2018. Evolution of the Natural Transformation Protein, ComEC, in Bacteria. Front Microbiol 9:2980.

57. Baker JA, Simkovic F, Taylor HM, Rigden DJ. 2016. Potential DNA binding and nuclease functions of ComEC domains characterized in silico. Proteins 84:1431-42.

58. Damke PP, Di Guilmi AM, Varela PF, Velours C, Marsin S, Veaute X, Machouri M, Gunjal GV, Rao DN, Charbonnier JB, Radicella JP. 2019. Identification of the periplasmic DNA receptor for natural transformation of Helicobacter pylori. Nat Commun 10:5357.

59. Lacks S, Neuberger M. 1975. Membrane location of a deoxyribonuclease implicated in the genetic transformation of Diplococcus pneumoniae. J Bacteriol 124:1321-1329. 
779 60. Puyet A, Greenberg B, Lacks SA. 1990. Genetic and structural 780 characterization of EndA. A membrane-bound nuclease required for 781 transformation of Streptococcus pneumoniae. J Mol Biol 213:727-738.

782 61. Dubnau D, Davidoff-Abelson R. 1971. Fate of transforming DNA following 783 uptake by competent Bacillus subtilis. I. Formation and properties of the

62. Patrick JE, Kearns DB. 2008. MinJ (YvjD) is a topological determinant of cell Galardini M, Cabal A, Peters JM, Hachmann AB, Rudner DZ, Allen KN, Typas A, Gross CA. 2017. Construction and Analysis of Two Genome-Scale Deletion Libraries for Bacillus subtilis. Cell Syst 4:291-305 e7. 
793

794

795

796

797

798

799

800

801

802

803

804

805

806

807
Table 1

Binding and uptake of rDNA by transformation mutants ${ }^{\mathrm{a}}$

\begin{tabular}{|l|l|l|l|l|l|l|l|l|}
\hline Mutant & \multicolumn{3}{|c|}{ No DNAase treatment } & \multicolumn{3}{c|}{ With DNAase treatment } \\
\hline & $\begin{array}{l}\text { With } \\
\text { signal }\end{array}$ & N & $\begin{array}{l}\text { Percent } \\
\text { With } \\
\text { signal }\end{array}$ & p-value & With & N & $\begin{array}{l}\text { Percent } \\
\text { Signal }\end{array}$ & p-value \\
& & & & & & $\begin{array}{l}\text { With } \\
\text { signal }\end{array}$ & \\
\hline$\triangle$ comEA & 12 & 99 & 12.1 & & 2 & 133 & 1.5 & \\
\hline Wild-type & 82 & 99 & 82.8 & $<0.00001$ & 92 & 108 & 85.2 & $<0.00001$ \\
\hline$\triangle$ nucA & 39 & 137 & 28.5 & & 13 & 126 & 10.3 & \\
\hline Wild-type & 88 & 138 & 63.8 & $<0.00001$ & 70 & 123 & 56.9 & $<0.00001$ \\
\hline nucA D98A & 45 & 114 & 39.5 & & 21 & 126 & 16.7 & \\
\hline Wild-type & 70 & 124 & 56.5 & $<0.009$ & 47 & 162 & 29.0 & 0.015 \\
\hline$\Delta$ comFA & 45 & 110 & 40.9 & & 32 & 104 & 30.8 & \\
\hline Wild-type & 86 & 110 & 78.2 & $<0.00001$ & 80 & 103 & 77.7 & $<0.00001$ \\
\hline comFA K15 & 82 & 136 & 60.3 & & 91 & 163 & 55.8 & \\
\hline Wild-type & 65 & 118 & 55.1 & 0.40 & 68 & 103 & 66.0 & 0.10 \\
\hline comEC518 & 66 & 107 & 61.7 & & 47 & 150 & 31.3 & \\
\hline Wild-type & 112 & 147 & 76.2 & 0.01 & 91 & 136 & 66.9 & $<0.00001$ \\
\hline
\end{tabular}

aData for each mutant was obtained in a separate experiment in mixed suspension with a wild-type control.

${ }^{\mathrm{b}}$ Determined with a two-tailed $\mathrm{z}$ test. 


\section{FIGURE LEGENDS}

810 Figure 1. Binding of tDNA and acquisition of DNAase resistance. Competent cells 811 expressing CFP (BD5810) or YFP (BD6011) from the comG promoter were 812 incubated separately with rDNA and samples were taken after (A) 2- and (B) 30813 minutes of incubation. The CFP samples were treated with DNAase and then 814 combined with YFP samples from the same time points. In panels A and B, images 815 are presented with superimposed rhodamine, CFP, YFP and phase contrast 816 channels as well as one showing only the rhodamine channel for clarity. Cells 817 associated with rDNA are indicated by arrows. The circled cell in panel A was 818 imaged by 3D deconvolution and volume reconstructed and views produced by 819 successive $90^{\circ}$ rotations are shown in panel C. In panel D volume reconstructed 820 images are shown for the single cyan (with DNAase) and yellow (no DNAase) cells, 821 circled in panel B. Panel E contains a 3D-deconvolved optical slice from the center 822 of a Z-stack, showing of a group of cells after DNAase treatment.

824 Figure 2. Binding and uptake in the comFA K152A mutant. Wild-type (YFP) and 825 mutant (CFP) cells were combined before 30 minutes incubation with rDNA. 826 Panels A and B show results for comFA K152E without and with DNAase 827 treatment respectively. In each panel the top and bottom rows show 9 each of 828 wild-type and mutant cells respectively. All the cells were taken from a single 829 representative microscope field and show all or nearly all of the rDNA-associated 830 cells in that field. In the cropped images of a given panel, rDNA signals were 831 enhanced identically but rDNA signal intensities cannot be compared between 832 panels $\mathrm{A}$ and B. Panel C shows volume reconstructed images of several 833 representative wild type (top row) and mutant (bottom row) cells. In each case a 834 single view is shown except for the adjacent images joined by brackets, which 835 show two views of the same cell related by $180^{\circ}$ rotations.

837 Figure 3. Binding and uptake in the $\triangle$ comEA mutant. Wild-type (YFP) and $\triangle$ comEA 838 cells were combined before incubation for 30-minutes with rDNA. Panel A shows 
839 the cells without DNAase treatment and B shows cells with treatment. In the top 840 images the rhodamine, CFP and YFP channels were merged and the bottom 841 images in A and B show only the rhodamine channel. The arrows indicate the 842 position of all the rhodamine signals. Panel C shows one aspect from a 3D 843 deconvolution of the CFP- and YFP-expressing cells circled in Panel A.

845 Figure 4. Binding and uptake in the nucA D98A mutant. Wild-type (YFP) and D98A 846 (CFP) mutant cells were made competent, combined, and after 15 minutes 847 incubation with rDNA were imaged without (panel A) and with DNAase (panel B). 848 In each of these panels the top and bottom rows show nearly all the wild-type and 849 mutant cells from a single field, respectively. The rhodamine signal was enhanced 850 before cropping the cells and the intensities of the rhodamine signal can be 851 directly compared. The YFP and CFP images were separately adjusted in each 852 image so as not to obscure the rDNA signal. The images in these two panels were 853 ordered by apparent rDNA signal strengths decreasing from left to right, to 854 facilitate comparisons of the mutant and wild-type cells. The boxed image in panel 855 B shows both a YFP- and a CFP-expressing cell.

857 Figure 5. Binding and uptake in the comEC518 mutant. Wild-type (YFP) and $858 \Delta$ comEC518 (CFP) cells were combined before 30-minutes incubation with rDNA. 859 The images were processed as described in the legend to Figure 4. Panel A shows 860 the cells without DNAase treatment and B shows cells with treatment. The single 861 image to the right of panel B shows two YFP cells and one CFP cell. The images in 862 these two panels were ordered by apparent rDNA signal strengths decreasing 863 from left to right, to facilitate comparisons of the mutant and wild-type cells. Panel 864 C shows selected volume reconstructed images of wild-type and comEC518 cells 865 selected from the same field, representing almost all the cells in the field. Only one 866 view is shown of each cell. Image intensities cannot be compared either within 867 panel C or with the images in panels A and B. 
869 Figure 6. Association of rDNA with YFP-ComEA without DNAase treatment. In 870 each group of four images (a-d in panel A and a-h in panel B), the top and bottom 871 pairs show views of a single cell, related by $180^{\circ}$ rotations. The left-hand images 872 in each group show the superimposed YFP, rhodamine and CFP channels, where 873 CFP delineates the cytoplasmic volume. The right-hand images show only the YFP 874 and rhodamine channels, for clarity. Panel A shows images collected after 1 875 minute incubation of the cells with rDNA and Panel B after 10 minutes.

876 
A

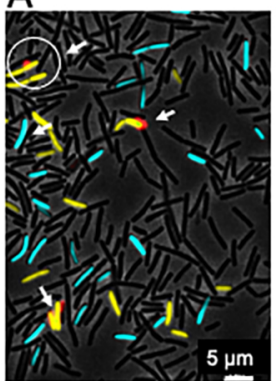

C

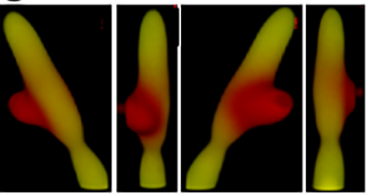

$\alpha$
B

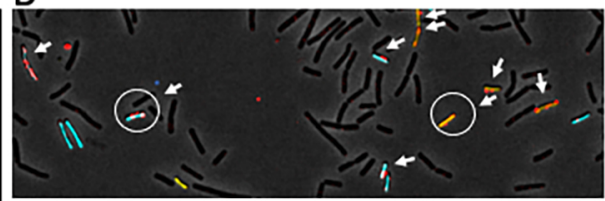

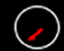

$5 \mu \mathrm{m}$

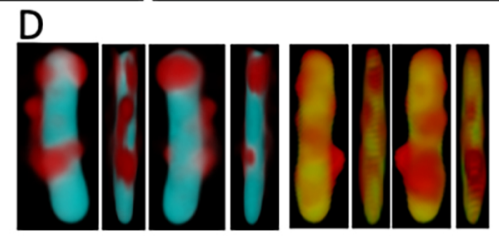

E

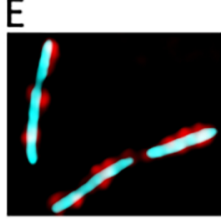



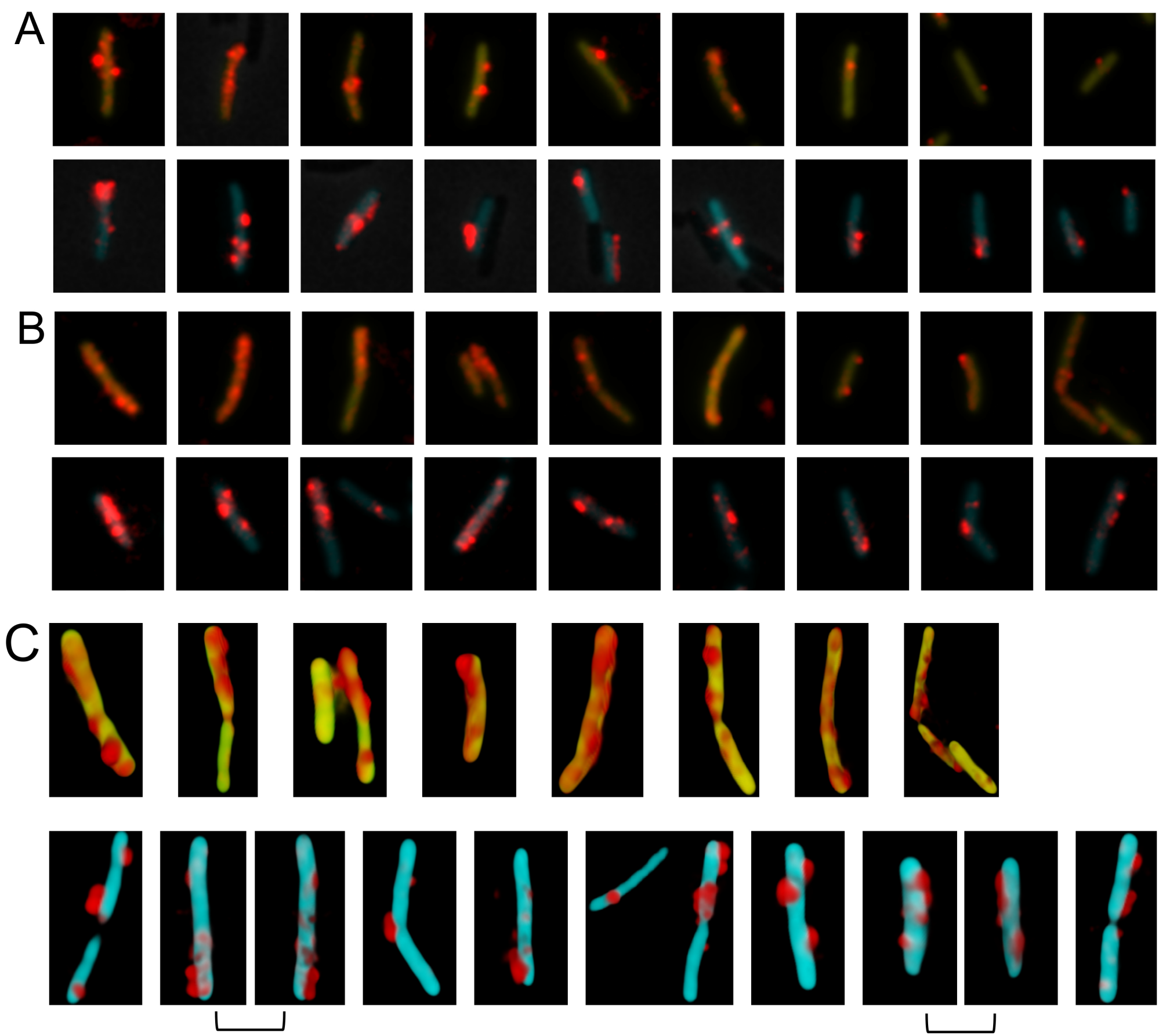


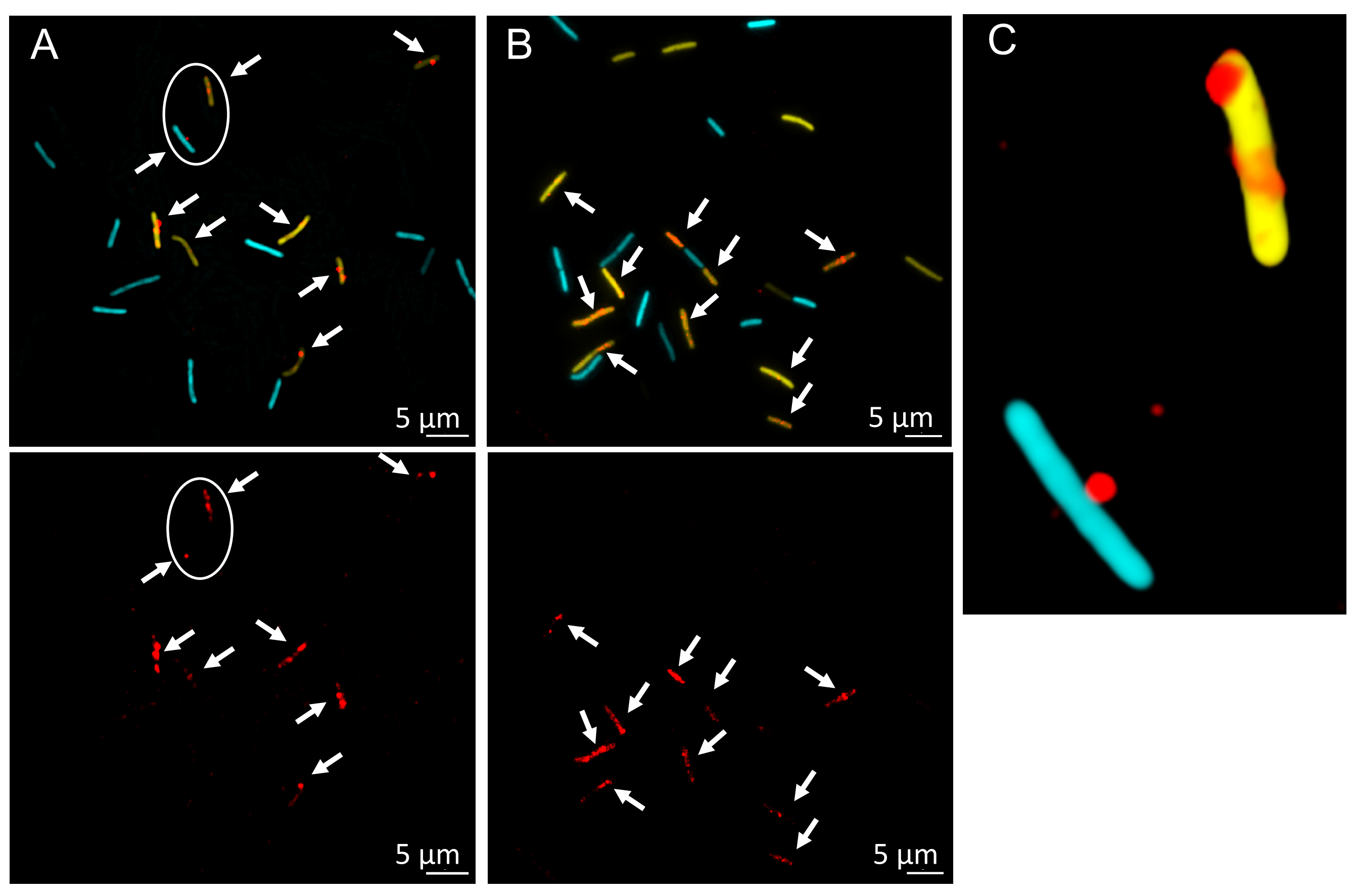




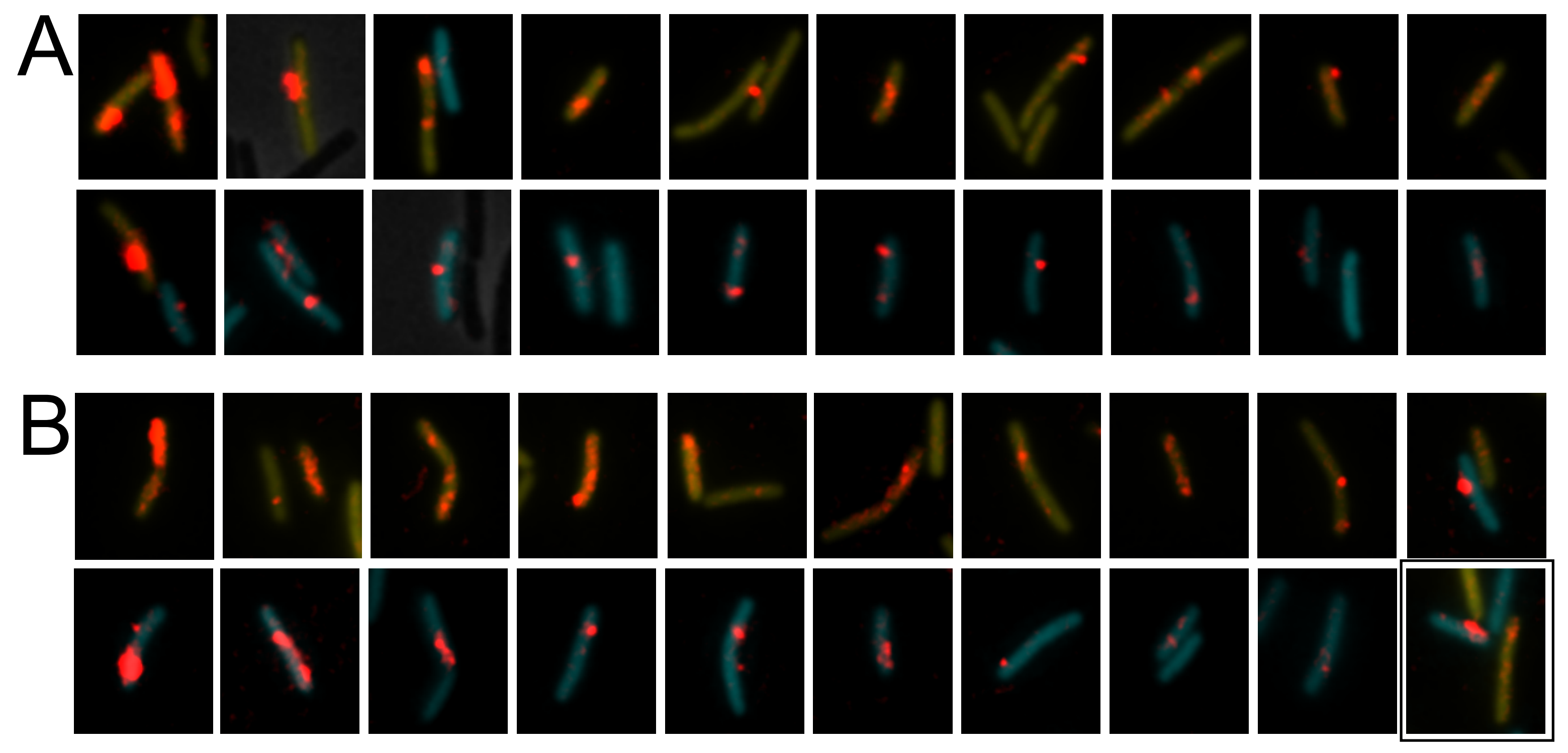




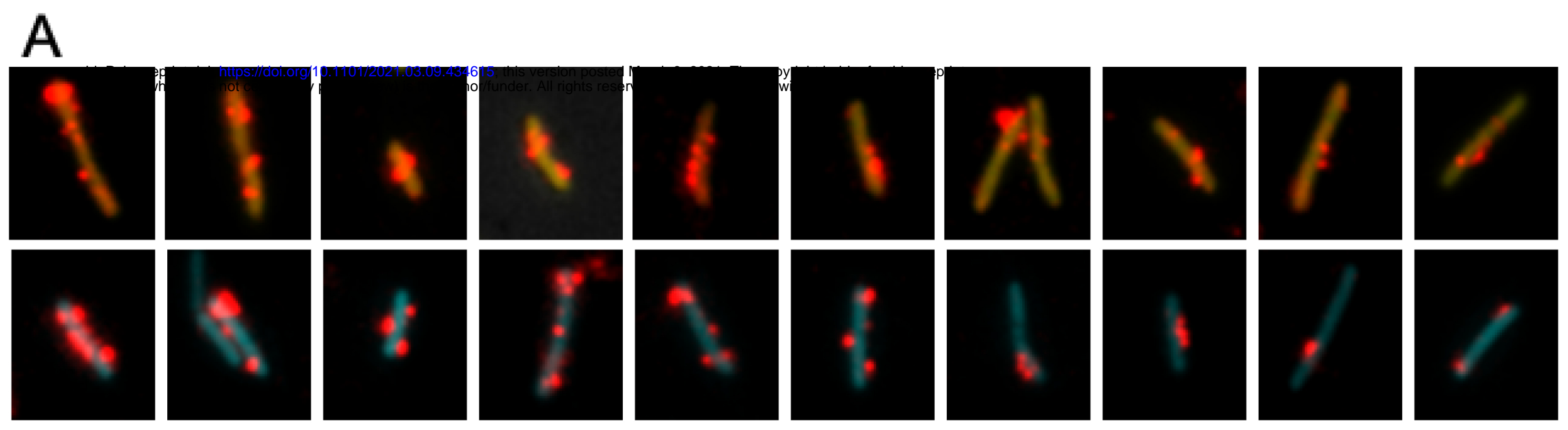

B

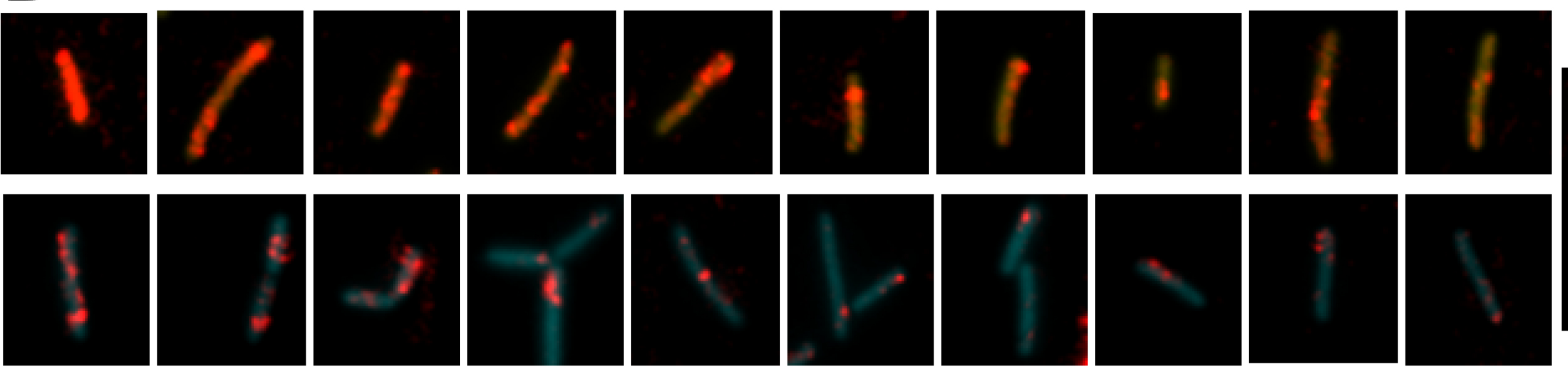

C

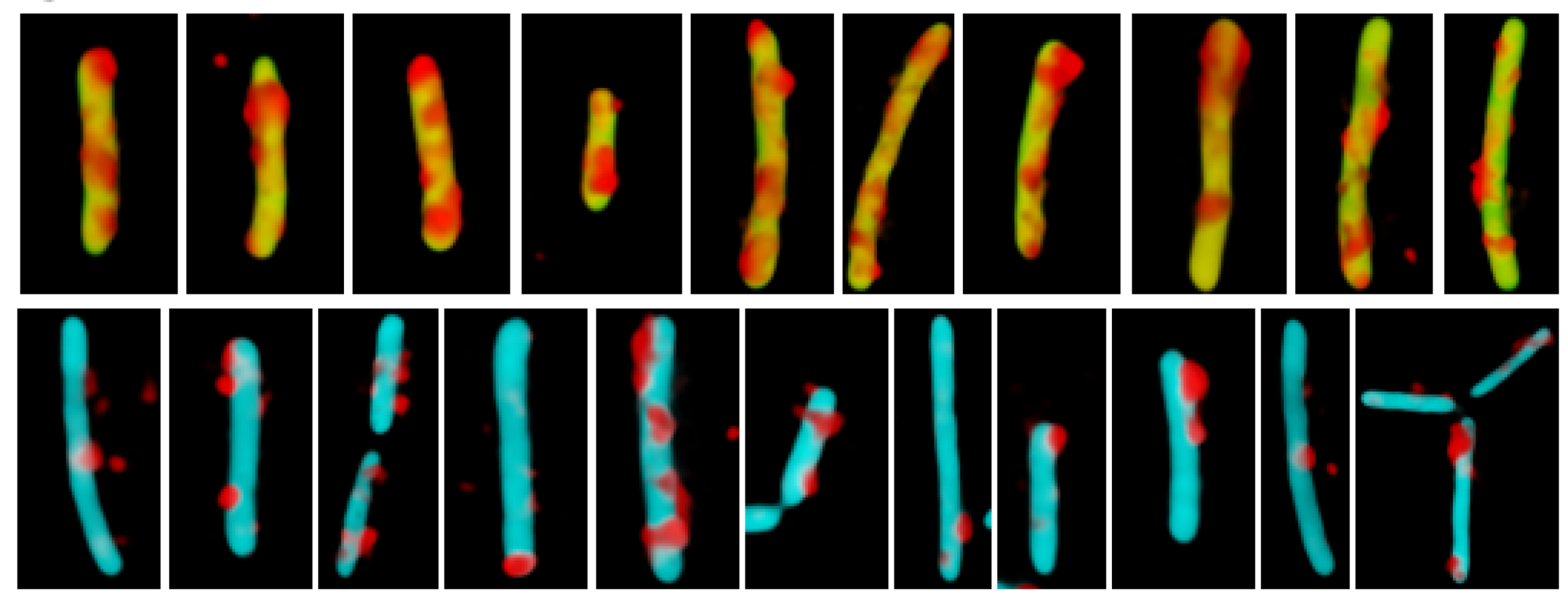




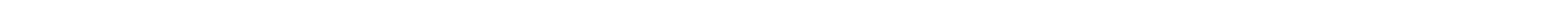


1

2

3

4
Table S1

Strains

\begin{tabular}{|c|c|c|}
\hline Strain $^{a}$ & Genotype & Source \\
\hline BD5810 & amyE::P $P_{G-} C F P(s p c)$ & $(48)$ \\
\hline BD6011 & $a m y E:: P_{G-} Y F P(s p c)$ & $(48)$ \\
\hline BD8739 & $\operatorname{trpC2} \triangle \operatorname{comEA}(\text { ery })^{b}$ & \\
\hline BD8800 & $a m y E:: P_{G-} C F P(s p c) \triangle c o m G A(e r y)^{b}$ & This work \\
\hline BD8802 & amyE:: $\operatorname{Pr}_{G-C F P}(s p c) \triangle c o m E A(e r y)^{b}$ & This work \\
\hline BD8805 & amyE:: $P_{G-} C F P(s p c) \triangle n u c A(e r y)^{b}$ & This work \\
\hline BD8883 & amyE::P $P_{G-C F P}(s p c) \triangle \operatorname{comC}(\text { ery })^{b}$ & This work \\
\hline BD8884 & amyE::P $P_{G-C F P}(s p c)$ comFA K152E & $(33)$ \\
\hline BD8911 & amyE::P $P_{G-} C F P(s p c) \triangle c o m F A\left(\right.$ ery) ${ }^{b}$ & This work \\
\hline BD8949 & $\begin{array}{l}\text { amyE:: } P_{G-C F P}(s p c) \text { yfp-comEA } \triangle \text { comEA } \\
(e r y)^{b}\end{array}$ & This work \\
\hline BD8951 & amyE::PG-CFP (spc) nucAD98A & This work \\
\hline BD8954 & $a m y E:: P_{G-} C F P(s p c)$ comEC-518 & (29) \\
\hline
\end{tabular}

5

6

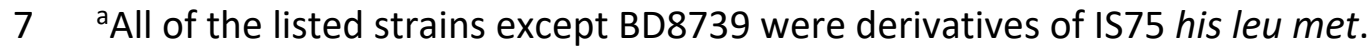

8

$9{ }^{b}$ These deletion mutations, constructed by Koo et al (63), were obtained from the

10 Bacillus Genetic Stock Center 
Table S2

12

Primers

14

\begin{tabular}{|c|c|}
\hline $\begin{array}{l}\text { Primer } \\
\text { number }\end{array}$ & Sequence $\left(5^{\prime}->3^{\prime}\right)$ \\
\hline 1 & AGAGGATCCCCGGGTACCGAGCTCGAATTCAAAAGGTATCGAGTAGCCAG \\
\hline 2 & CTCCTTTACTCATATTCATCGTGCATGTTCC \\
\hline 3 & ATGCACGATGAATATGAGTAAAGGAGAAGAAC \\
\hline 4 & TCAACCAATTCATTTTTGATAGTTCATCCATGC \\
\hline 5 & GGAAACAGCTATGACCATGATTACGAATTCACTTTACTGTAATGGAAG \\
\hline 6 & ACTATACAAATAAATGAATTGGTTGAATCAGC \\
\hline
\end{tabular}

15 
16 Figure S1. Western blots for transformation proteins in mutant strains. All of the

17 mutants were deletions except for comEC518 which is a transposon insertion near the

18 beginning of the coding sequence. In all cases the relevant knockout mutant was used

19 as a negative control for the antiserum. There is a consistent cross-reacting band at

20 the position of the ComEC signal. For most of the gels a loading control is shown using

21 anti-elongation factor G (EFG) antiserum, a kind gift from J. Dworkin. In panel D, the

22 cross-reacting band just below the ComEA signal serves as a loading control. In panel

$23 \mathrm{E}$, a cross-reacting band is visible in the comEC518 lanes at the position of ComEC (29).

Figure S2. DNA binding in $\triangle \operatorname{comGA}$ and $\triangle \operatorname{comC}$ strains. $\triangle \operatorname{com} G A(\mathrm{~A})$ and $\triangle \operatorname{com} C$ (B)

26 strains, expressing CFP, were combined with transformed wild-type bacteria

27 expressing YFP. Transformation was for 30 minutes without DNAase treatment. The

28 arrows indicate all the cells with detectable rDNA signals. The CFP labeled $\triangle$ comGA

29 cells are slightly filamented (Hahn J, Tanner AW, Carabetta VJ, Cristea IM, Dubnau D.

30 2015. Mol. Microbiol 97:454-71).

32 Figure S3. Binding and uptake in the $\triangle$ comFA mutant. Wild-type (YFP) and mutant 33 (CFP) cells were combined before 30-minutes incubation with rDNA. Panels A and B

34 show results for $\triangle$ comFA without and with DNAase treatment respectively. In each 35 panel the top and bottom rows show 9 each of wild-type and mutant cells 36 respectively. All the cells were taken from a single representative microscope field 37 and show all or nearly all of the rDNA-associated cells in that field. In the cropped 38 images of a given panel, rDNA signals were enhanced identically but rDNA signal 39 intensities cannot be compared between panels $A$ and $B$.

41 Figure S4. Binding and uptake in the $\triangle n u c A$ mutant. Wild-type (YFP) and mutant (CFP) 42 cells were combined before 15-minutes incubation with rDNA and imaged without 43 (panel A) and with DNAase (panel B). In each of these panels the top and bottom rows 44 show nearly all the wild-type and mutant cells from a single field, respectively. The 
45 rhodamine signal was enhanced before cropping the cells and the intensities of the

46 rhodamine signal can be directly compared. The YFP and CFP images were separately

47 adjusted in each image so as not to obscure the rDNA signal. The images in these two

48 panels were ordered by apparent rDNA signal strengths decreasing from left to right,

49 to facilitate comparisons of the mutant and wild-type cells. The boxed images both a

50 YFP- and a CFP-expressing cell.

51

52 Figure S5. Volume reconstructions of the $\triangle$ nucA (panel A) and nucA D98A (panel B)

53 mutant strains. Wild-type (YFP-expressing) and mutant (CFP-expressing) cells are

54 shown in each panel for comparison.

55

56

57

58 


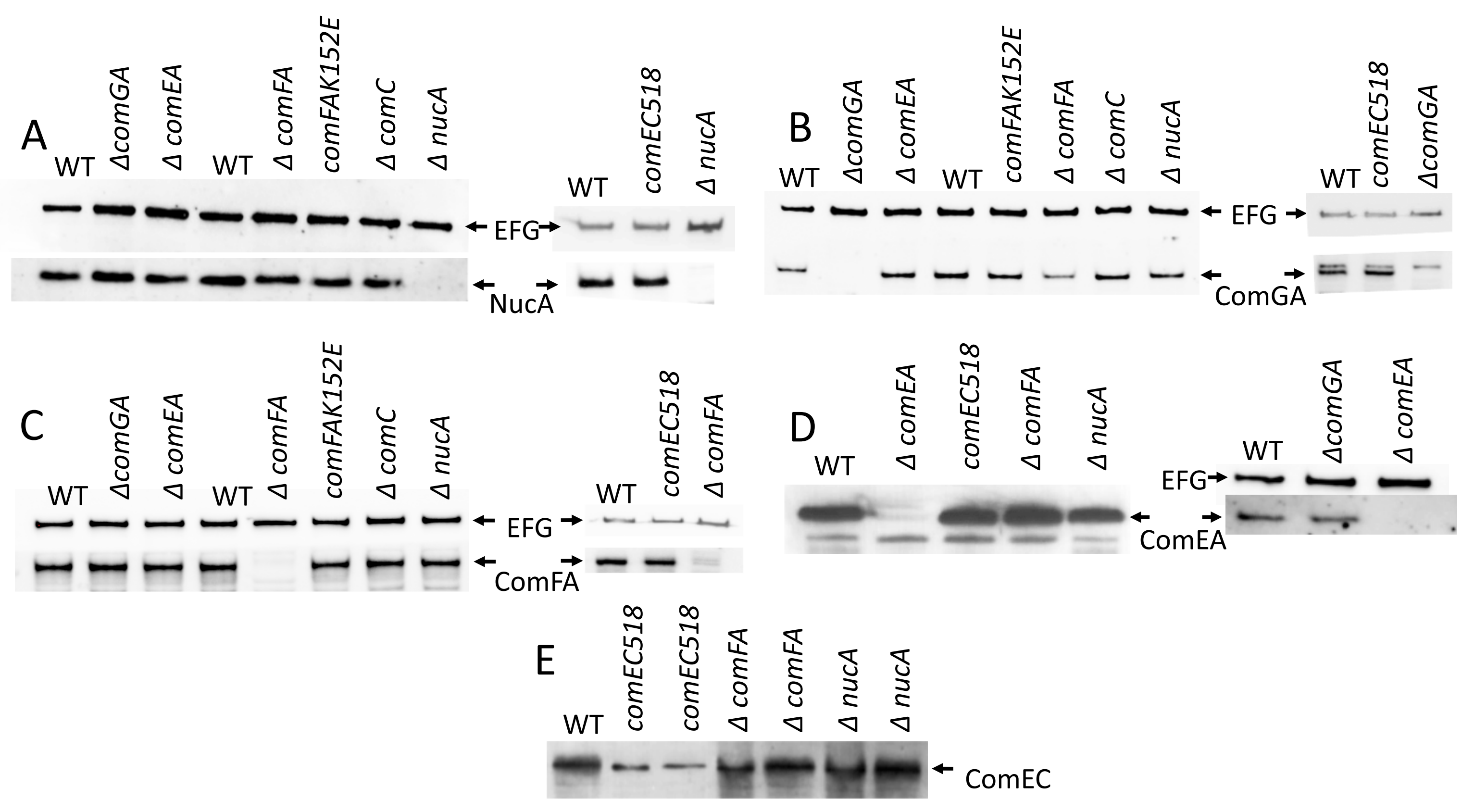




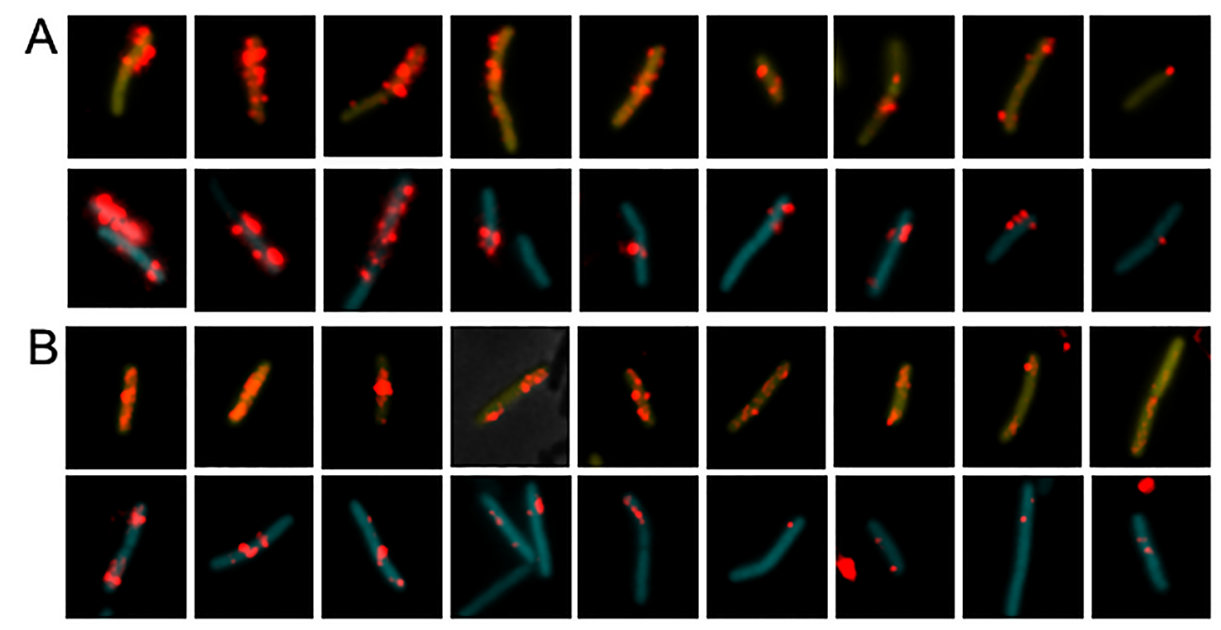




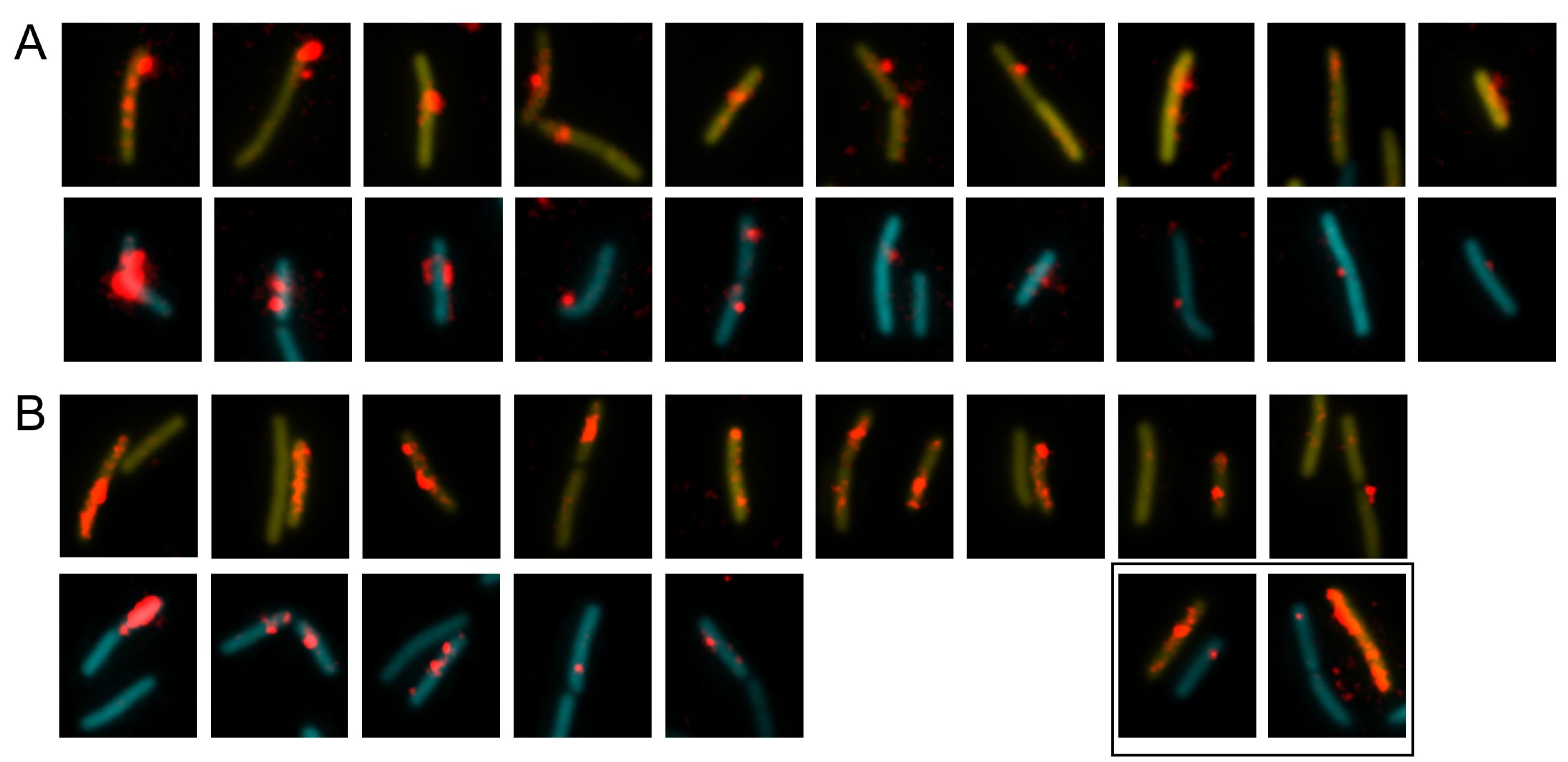

$A$

B 


\section{A}
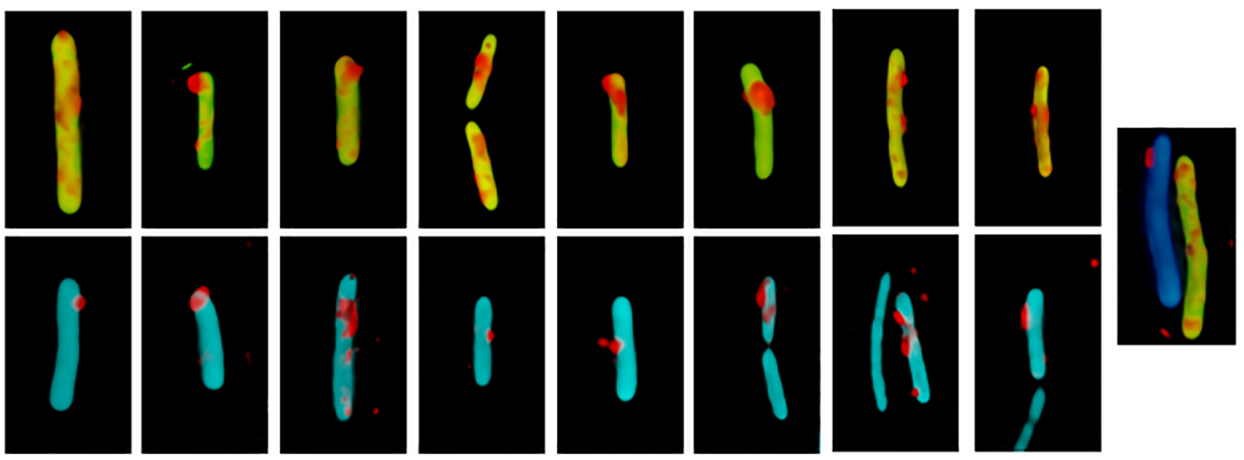

B
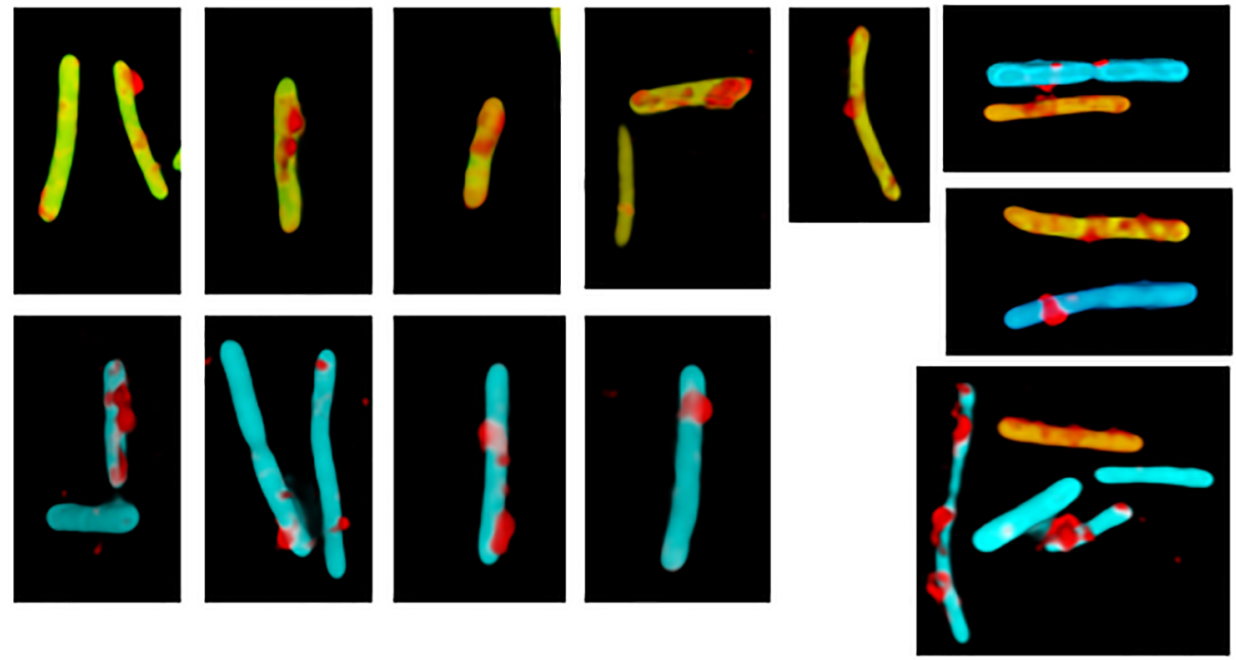